\title{
Effects of Caching on the Performance of DSR Protocol
}

\author{
Narinderjeet Kaur ${ }^{1}$, Maninder Singh ${ }^{2}$ \\ ${ }^{(1}$ Punjabi University, Department of Computer science, Patiala, India \\ ${ }^{2}$ Punjabi University, Department of Computer science, Patiala, India
}

\begin{abstract}
Route cache strategy is important in an on demand routing protocol in wireless mobile ad hoc networks. In on demand routing protocol the route discovery mechanism is used to transmit packets from one mobile node to other. In order to avoid such route discovery mechanism each time when the packet is transmitted, the route caching technique is used. Route caching is the major approach to decrease the flooding of the network by avoiding the route discovery operation as much as possible. Because the frequently use of route discovery mechanism is very costly in terms of delay and bandwidth consumption which can cause congestion and long delay. Therefore the efficient caching strategies have the great impact on the performance of the DSR routing protocol. This paper, presented the effect of the cache expiry time and cache capacity on the performance of the DSR routing protocol. The simulation results indicate that the cache capacity can indirectly affect the performance of the routing protocol. The performance of the DSR protocol decreases if we increase the cache size, because increase in cache size cause stale routes in the cache. The stale routes are generated due to the mobility of the nodes. To reduce or remove the stale routes cache expiry time is used.
\end{abstract}

Keywords-MANET, DSR, Route Cache, Cache Expiry time, Cache Size.

\section{INTRODUCTION}

Mobile ad hoc network is the important topic of research. Mobile ad hoc network is collections of wireless nodes that can allow people and devices to communicate with each other without help of an existing infrastructure. Each device in a MANET is free to move independently in any direction, and will therefore change its links to other devices frequently. In MANET the mobile node can be work as a router or host. Because they are independent of any infrastructure therefore are used in military communication and rescue operations where the development of an infrastructure is neither feasible nor cost effective. There are three categories of Mobile ad hoc network routing protocols, which are Proactive (or periodic) routing protocols (DSDV, OLSR), reactive (or on demand) routing protocols (DSR, AODV, TORA) and hybrid routing protocols. Proactive routing protocols maintain the routes independent of the traffic pattern. On the other hand reactive routing protocols determine the route only if needed. Hybrid routing protocol (ZRP) combined the advantages of proactive and reactive routing. Reactive routing protocols have less overhead since routes are maintained only if they are needed but proactive routing protocols have higher overhead due to continuous route updating.

\section{DSR PROTOCOL}

Dynamic Source Routing (DSR) is a reactive, flexible and simple protocol. DSR is on demand routing protocol and uses the concept of source routing instead of routing tables like other protocols. When source node send packets to the destination node, the different packets may follow the different routes, even they have same source and destination. DSR allow the packets to be travel loop-free and avoid the need for updating routing information in the routing tables periodically that is required in table-driven approach. Therefore DSR routing protocol reduce the bandwidth consumption by the control packets in ad hoc mobile networks. The major difference between this and the other on-demand routing protocols is that it is beacon-less and hence does not require periodic hello packet (beacon) transmissions, which are used by a node to inform its neighbors of its presence. The DSR protocol is composed of two main mechanisms Route Discovery and Route Maintenance.

\subsection{Route Discovery}

When any mobile node or source node want to send a packet to any other node known as destination node and does not know a route to it, the source node initiates the route discovery. To initiate the Route Discovery, the source node floods a "Route Request" with a unique ID as a single local broadcast packet. Each node appends its own identifier when forwarding the route request. When some intermediate node receives this Route Request, at first it determines whether it has seen the Route Request or not. If the node has already seen the Route Request earlier, it will discard the packet; otherwise it will check its Route Cache whether there is a 
route to the destination of the packet. If it has the route to target in its routing cache, it returns a "Route Reply" to the initiator of the Route Discovery, giving a copy of the accumulated route record from the Route Request; otherwise it transmits the Route Request until the Route Request is received by the target .

\subsection{Route maintenance}

DSR protocol implements the route maintenance mechanism while communicating the packets from source to destination. But when the communication link between the source and the destination is broken or else a change in network topology is noticed. It will lead to failure of the communication between source node and destination node. In this scenario DSR protocols uses the route maintenance mechanism, to detect any other possible known route towards the destination to transmit data. If the route maintenance fails to find an alternative known route to establish the communication then it will invoke the route discovery to find the new route to destination.

The Dynamic Source Routing (DSR) protocol is based on source routing, which means that the originator of each packet determines an ordered list nodes through which packet must pass while traveling to the destination. Flooding method is used in DSR protocol for route discovery, which cause the traffic load on the network. To decrease the flooding of the network Route Caching is used and the overall performance of the network can be significantly increased.

\section{ROUTE CACHING IN DSR PROTOCOL}

Route caching in DSR protocol is used to store the routes that have learned from the source node and to avoid unnecessary route discovery operation each time a data packet is to be transmitted. Because that reinitiating of a route discovery mechanism in on demand routing protocols is very costly in term of delay, battery power, and bandwidth consumption due to flooding of the network, which can cause long delay before the first data packet sent. The performance of DSR protocol mainly depends on an efficient implementation of route cache.

\subsection{Cache Capacity}

The capacity of a route cache is another important area of choice in designing a caching strategy for ondemand routing protocols. Cache capacity or cache size has a great impact on the performance of the DSR routing protocol. Cache capacity can be divided into two halves: first half called Primary Cache, represents paths that are used by the current nodes and second half called Secondary Cache, and represents paths that have not yet used. When a source node attempts to add new path that have not yet been used into the cache, old path in the secondary cache are removed due to limitation of the cache size. We consider the effects of different limits on the capacity of path caches in terms of the number of individual paths it can store.

\subsection{Cache Timeout}

As with cache capacity, cache timeout policy introduces a number of design choices to consider in a caching strategy. Two types of cache timeout approaches are used: static timeout approach and adaptive time out approach. In static time out approach same timeout value is used for each link that is stored in the cache. Each link or path is removed from the cache after that specific value of time. But in adaptive time out approach the links or paths are removed based on the stability of the link. The link is removed from the cache which is least used. Cache timeout is an important factor because it also removes the stale routes present in the route cache.

\section{RELATED WORK}

Shobha K.R. et al. [6] presents an analysis of the effect of intelligent caching in non clustered network, using on demand routing protocol. In Intelligent Caching technique a node not only saves the path discovered during route discovery for itself but also for others who are located close to it. This technique reduces the number of route request packets unnecessarily circulating in the network, when the path it requires is present in its neighborhood. This technique drastically increase the available memory for caching the routes discovered without affecting the performance of the DSR protocol in any way except for a small increase in end to end delay.

To avoid performing route discovery mechanism before each data packet is sent, DSR needs to formerly cache the routes discovered. T.C. Huang et al. proposed two mechanisms to improve cache performance of DSR: RERR-Enhance mechanism and hierarchical link cache structure as described in [8], the average end to end delay and routing overhead is improved with these mechanisms.

The route cache is used in DSR protocol to store all the routes are learned from the source node and to avoid unnecessary route discovery process. Naseer Ali Husieen et al. described in [3] with high 
mobility situations and high load network traffic stale routes will be generated. These stale routes can mainly affect the performance of DSR protocol which cause long delay, increase the packet loss, increase the overhead and reduce the efficiency of protocol. Therefore efficient mechanism for updating the route in the cache of DSR protocol is needed.

Shukla et al. [7] proposed new route cache maintenance in DSR protocol in order to improve the performance of the DSR protocol. This was achieved by allowing nodes to learn about the route caches of neighbors' nodes, instead of sending route error packets to the source, due to link failure, before forwarding the packet to the destination.

\section{SIMULATION FRMEWORK}

Simulation is done using OPNET Modeler 14.5 simulator tool. OPNET 14.5 is a network modeling and simulation software tool. OPNET Modeler 14.5 executes four phases in order to complete a simulation process. These phases are: modeling of the network, choosing the required parameters, running the simulation of the created model, and viewing and analysis of the results.

\section{SIMULATION PERAMETERS AND RESULTS}

Simulation was done for a campus sized network. The mobile nodes have a common traffic source generated by a MANET traffic generator and they are WLAN mobile clients transmitting a power of $0.1 \mathrm{~W}$ and a data rate of $11 \mathrm{Mbps}$. The traffic generator also has a data rate of $11 \mathrm{Mbps}$ but a transmitting power of $0.5 \mathrm{~W}$. Application configuration is used to supporting applications as HTTP (heavy browsing). Profile configuration is used for configuring the user profiles and objet profiles. Mobility configuration is used configuring the objects. Random waypoint mobility model was used for the simulation. This model ensures that the mobile nodes are configured with mobility status.

TABLE 1 Simulation Parameters

\begin{tabular}{|c|c|}
\hline Simulation Area & $700 \times 700 \mathrm{~m}$ \\
\hline Data Rate & $11 \mathrm{Mbps}$ \\
\hline Operational Mode & $802.11 \mathrm{~b}$ \\
\hline Routing Protocol & DSR \\
\hline Start Time & $20 \mathrm{sec}$ \\
\hline Pause Time & $0.1 \mathrm{Wec}$ \\
\hline Transmit Power & Constant \\
\hline Bit rate & Uniform(140,160) \\
\hline Packet Size(bits) & Random waypoint \\
\hline Mobility Model & $10 \mathrm{~m} / \mathrm{s}$ \\
\hline Nodes speed & $5 \mathrm{~min}$ \\
\hline Simulation time & \\
\hline
\end{tabular}

We consider the different scenarios having varying cache size and analyze the results for each scenario. Simulation is done for 20,30 and 80 nodes. For each scenario we set the DSR parameters and cache parameters such as cache timeout and cache size or capacity. The simulation results show the effects of caching parameters on the performance of DSR protocol. 


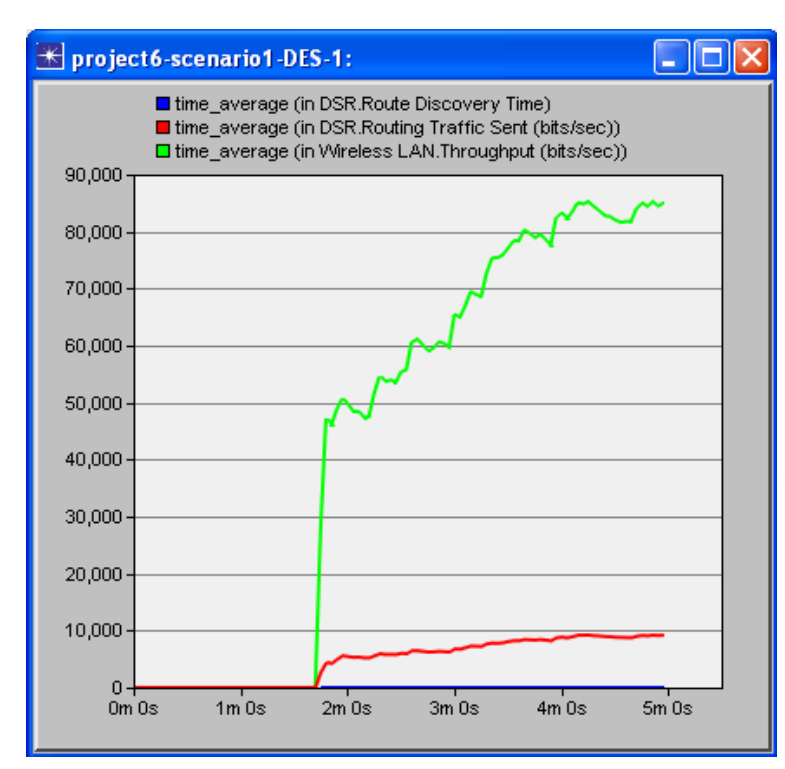

Fig.1 performance of DSR protocol for 20 nodes having cache

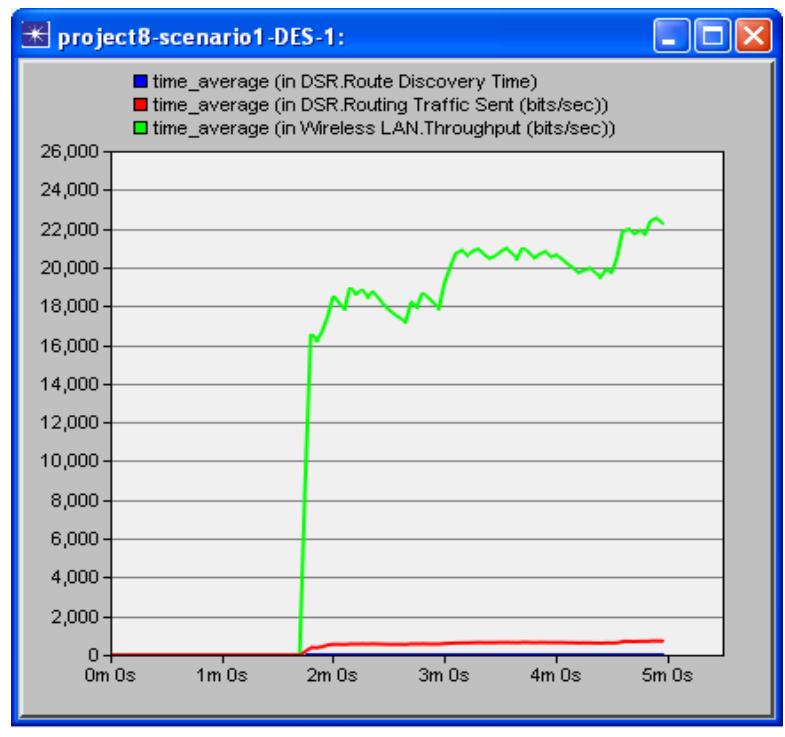

Fig.2 Performance of DSR protocol for 20 nodes having cache size 10 and cache expiry time 10 size 15 and cache expiry time 10

Figure 1 and 2 shows the results for 20 nodes with cache size 10 routes, cache expiry time 10 seconds and cache size 15 routes and cache expiry time 10 seconds respectivelly. Green line indicates the throughput, red line ndicates the traffic overhead and blue line indicates the route discovery time. Iin figure 1 the value of throughput is nearabout 85,000 but in figure 2 the value is aproximate 22,000. Terefore throughput decreases from 85,000 to 22,000 , that mean if the cache size increases the throughput decreases, because due to the mobility of the nodes the stale routes are generated in the cache which decreases the throughput. The traffic overhead increases to its maximum value and then becomes constent as red line indicates in figure 2.

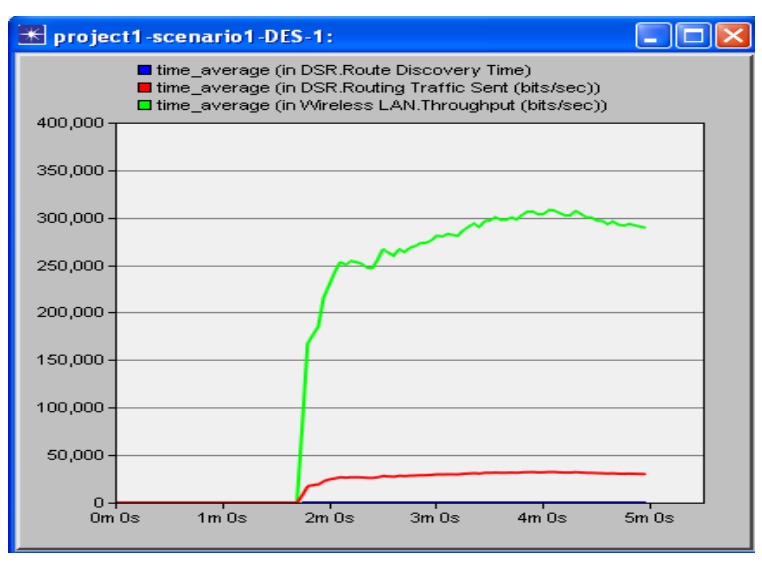

Fig. 3 Performance of DSR protocol for 30 nodes having cache size 10 and cache expiry time 10

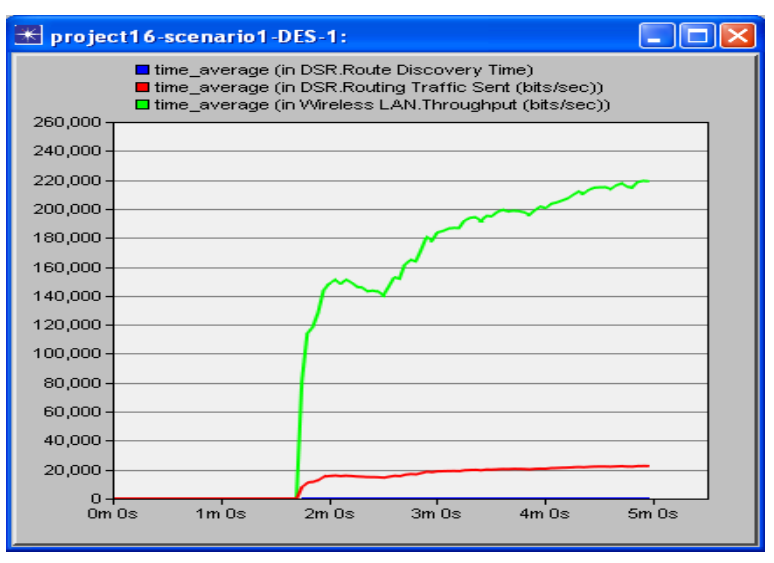

Fig.4 Performance of DSR protocol for 30 nodes having cache size 15 and expiry time 10 


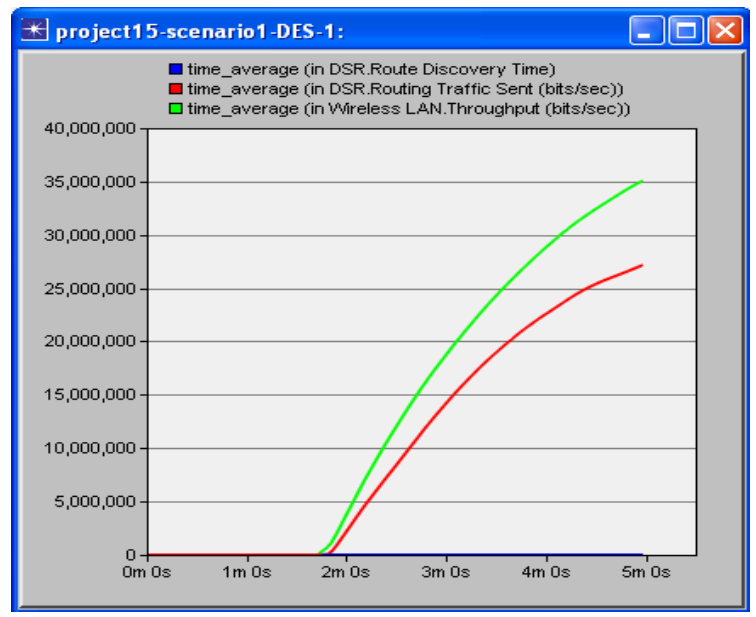

Fig.5 Performance of DSR protocol for 80 nodes having cache size 10 and cache expiry time 10

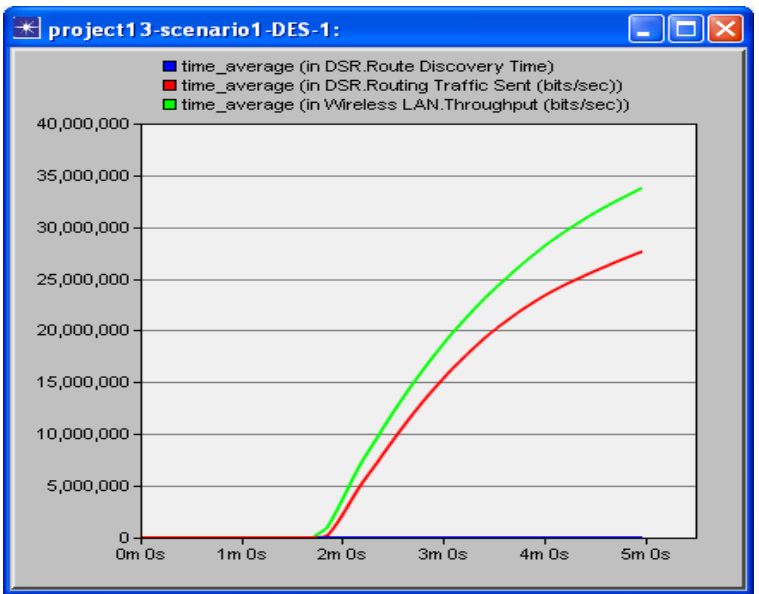

Fig.6 Performance of DSR protocol for 80 nodes having cache size 15 and cache expiry time 10

Figure 3 and 4 shows the results for 30 nodes and figure 5 and 6 shows the results 80 nodes. Results from these figures shows that with increase in the cache size, throughput decreases. The value of throughput is approximate $300,000 \mathrm{bits} / \mathrm{sec}$ in fig. 3 but decreases to 220,000 as shown in fig. 4 and throughput is 35,000,000 for 80 nodes having cache size 10 and expiry time 10 (fig. 5), but reduces to 34,000,000 as shown in fig. 6.Red line indicate the routing traffic overhead which shows that routing traffic overhead increases as the number of nodes increases within the network as shown in figure 5 and 6.

\section{CONCLUSION}

Route caching is the major approach to decrease the flooding of the network by avoiding the route discovery operation due to this congestion and long delay are also decreases. Therefore the efficient caching strategies have the great impact on the performance of the DSR routing protocol. In general, our intuition was that the larger the capacity of a cache, the better the routing protocol should perform, since it is able to keep a more complete set of routes. However, smaller cache size actually can have an indirect effect in improving performance. By increasing the cache capacity, throughput decreases because of stale routes that are generated due to the mobility of the nodes. Cache timeout is used to reduce or remove the stale routes.

\section{REFERENCES}

[1] Fenglien Lee, Carl T. Swanson, Jigang Liu, "Efficient On-Demand Cache Routing for Mobile Ad Hoc Networks", $2^{\text {nd }}$ IEEE International Conference on Digital Object Identifier, ICCSIT, pp. 167-171, 2009.

[2] Md. Golam Kaosar et al, “ Performance Improvement of Dynamic Source Routing Protocol Considering the Mobility Effect of Nodes in Cache Management”, IEEE International Conference, vol.6, 2006.

[3] Naseer Ali Husieen et al, "Route Cache Update Mechanisms in DSR Protocol", International Conference On Information and Network Technology IPCSIT vol.4, pp.136-141, 2011.

[4] P. Kuppusamy et al."A Review of Cooperative Caching Strategies in Mobile Ad hoc Networks", International Journal of Computer Applications (0975-8887), vol.29, pp.22-26, 2011.

[5] Sangeeta Biswas et al, " Study of DSR Protocol in Mobile Ad hoc Network", International Conference on Information and Network Technology IPCSIT vol.4, 2011.

[6] Shobha K.R. and K. Rajanikanth, "Intelligent Caching in On Demand Routing Protocol for Mobile Ad Hoc networks", World Academy of Science, Engineering and Technology 59, pp 413-420, 2009.

[7] Shukla, "Ensuring Cache Freshness in On-demand Routing Protocols for Mobile Ad Hoc Network: A Cross-layer Framework," 4th IEEE Costumer Communications and Networking Conference (CCNC), pp. 264-268, 2007.

[8] Tsung-Chuan Huang, Chi-Chen Chan, "Caching Strategies for Dynamic Source Routing in Mobile Ad Hoc Networks", IEEE Communication Society WCNC , pp. 4242-4246, 2007.

[9] Xin Yu, "A Distributed Adaptive Cache Update Algorithm for the Dynamic Source Routing protocol”, IEEE Transactions on Mobile Computing, vol. 5, pp. 609-626, Jun. 2006.

[10] Yih- Chun Hu, David B.Johnson, " Caching Strategies in On-Demand Routing Protocols for Wireless Ad Hoc Networks ”, Sixth Annual International Conference on Mobile Computing and Networking, pp- 6-12, August 2000. 